\title{
O cultivo da macieira na Itália: porta-enxertos, cultivares, adubação e irrigação
}

\author{
Giovambattista Sorrenti ${ }^{1}$ \\ Adamo Domenico Rombolà ${ }^{1}$ \\ Giuseppe Garcea ${ }^{1}$ \\ André Z. De Pieri ${ }^{1}$ \\ Duilio Porro ${ }^{2}$ \\ Gustavo Brunetto ${ }^{3 *}$ \\ Alcione Miotto ${ }^{4}$ \\ Djalma Eugênio Schmitt ${ }^{5}$ \\ Luciano Colpo Gatiboni ${ }^{5}$ \\ ${ }^{1}$ Università di Bologna, Bologna, Italia \\ ${ }^{2}$ Istituto Agrario di San Michele all'Adige, San Michele, Trento, Italia \\ ${ }^{3}$ Universidade Federal de Santa Catarina, Centro de Ciências Agrárias \\ CEP 88034-000, Florianópolis - SC, Brasil \\ ${ }^{4}$ Universidade Federal de Santa Maria, Santa Maria - RS, Brasil \\ ${ }^{5}$ Universidade do Estado de Santa Catarina, Lages - SC, Brasil \\ * Autor para correspondência \\ brunetto.gustavo@gmail.com
}

Submetido em 02/02/2012

Aceito para publicação em 21/06/2012

\section{Resumo}

A Itália é um dos principais países produtores de maçãs na Europa, destinada principalmente ao consumo in natura no mercado nacional e internacional. A produção e a qualidade dos frutos é dependente da cultivar, do porta-enxerto e das práticas de manejo, como a adubação e a irrigação adotado no pomar. Esta revisão tem por objetivo reportar as principais cultivares e porta-enxertos de macieira e o manejo da adubação e irrigação e suas atualizações em pomares de macieira da Itália. Os programas de melhoramento genético nesse país envolveram a seleção de cultivares e porta-enxertos de macieira que permitem a obtenção de altas produtividades e frutos de qualidade exigida pelo mercado consumidor. No manejo da adubação e irrigação, os nutrientes e a água têm sido fornecidos em quantidades próximas a real necessidade das plantas, proporcionando nutrição adequada, produção satisfatória e frutos de boa qualidade, além de evitar, sempre que possível, as perdas de nutrientes e água no ambiente.

Palavras-chave: Malus domestica; Nutrição mineral; Qualidade de fruto

\section{Abstract}

Apple tree production in Italy: rootstocks, cultivars, fertilization, and irrigation. Italy is one of the main apple producers in Europe, primarily intended for fresh consumption, both in the domestic and foreign 
markets. Fruit yield and quality depends on the cultivar, rootstock, and management practices, such as the fertilization and irrigation adopted in the orchard. This review aims at reporting the main apple cultivars and rootstocks, the management of fertilization and irrigation, as well as their adaptation to apple tree orchards in Italy. The programs for genetic improvement carried out in this country involved the selection of apple tree cultivars and rootstocks which enable a high fruit yield and quality, in order to meet the requirements from the consumer market. In the fertilization and irrigation management, nutrients and water are supplied in amounts next to the actual need of the plants, providing an adequate nutrition, a satisfactory yield, and high quality fruits, besides preventing, whenever possible, nutrients and water losses in the environment.

Key words: Fruit quality; Malus domestica; Mineral nutrition

\section{Introdução}

A Itália possui uma das maiores áreas cultivadas com a macieira (Malus domestica) da Europa e produz, aproximadamente, dois milhões de toneladas de maça por ano. Os frutos na sua maioria são destinados ao consumo in natura, especialmente no país, onde são consumidos, em torno de $17 \mathrm{~kg}$ de maçã por pessoa a cada ano, que é um dos maiores consumos per capita do fruto no mundo (DALPIAZ, 2007). As maiores áreas cultivadas com a macieira são encontradas nas regiões do Veneto, Friuli-Venezia Giulia e Trentino-Alto Adige (Figura 1), onde são produzidos, aproximadamente,
80\% da maçã nacional (Tabela 1). Além dessas regiões, o cultivo é expressivo na região da Emilia-Romagna e na Campania. Normalmente, em todas as regiões, os pomares de macieira apresentam longevidade média economicamente viável de 15 anos e são instalados em pequenas áreas nas propriedades, que podem possuir relevo com topografia plana, mas boa parte dos pomares de macieira italianos é encontrada em montanhas ou próximas a elas, como na região do Trentino-Alto Adige. Nestes locais com maior altitude, o microclima particular garante produções qualitativamente melhores e melhor exposição dos frutos à radiação solar, que contribue

FIGURA 1: Localização geográfica das províncias na Itália.

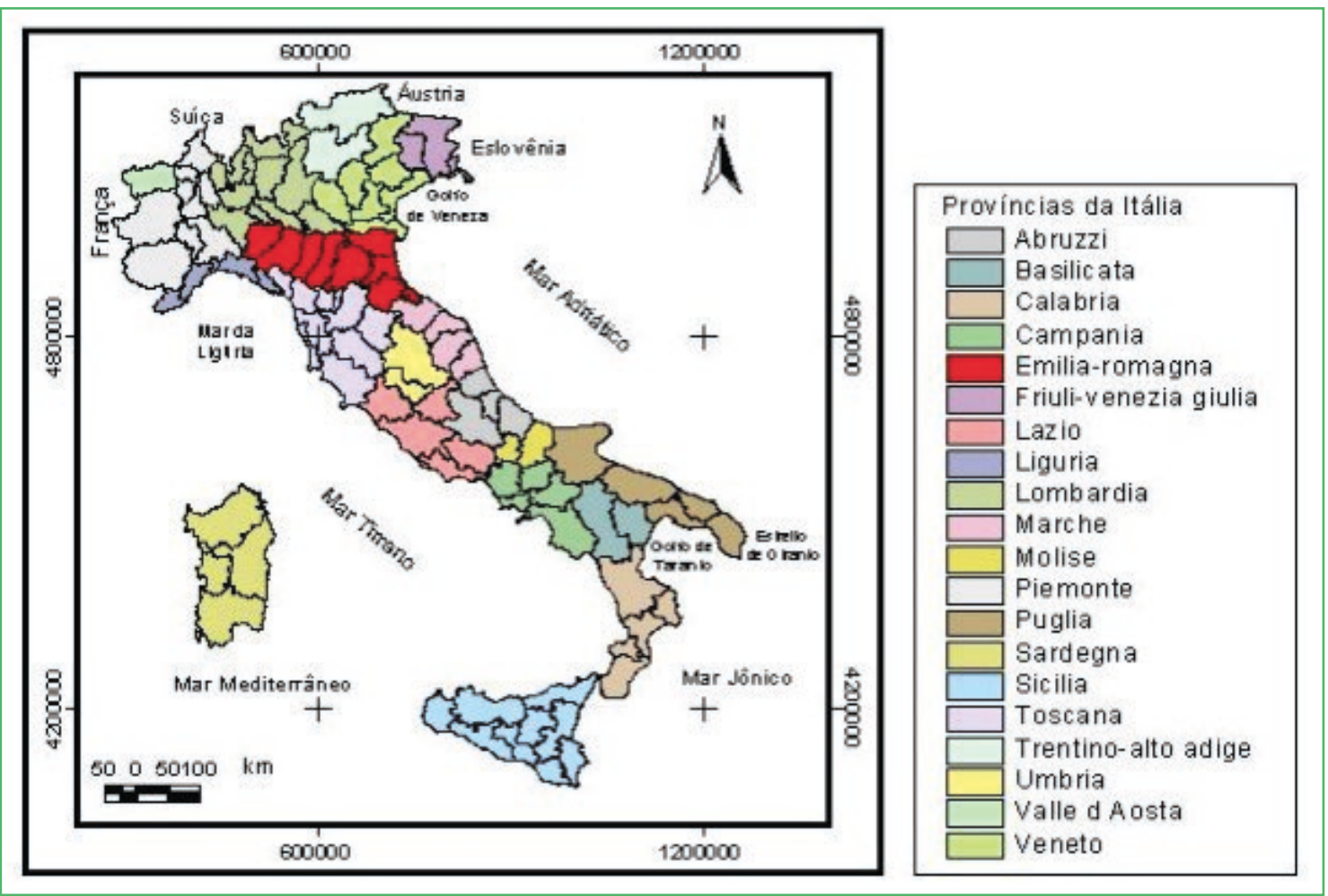


para acentuar a cor vermelha intensa e um equilíbrio natural entre ácidos e açúcares, salientando o sabor e o aroma (FERRANDINO et al., 1999; LA IACONA; GIACALONE, 2006).

Em todos os sistemas de produção da macieira na Itália têm sido observado avanços tecnológicos, como na escolha de cultivares mais apropriadas a cada região e com frutos mais vistosos, uso de porta-enxertos selecionados às diferentes situações e no manejo da adubação e da irrigação nos pomares, com maior preocupação ambiental. Como o Brasil é um expressivo produtor de maçãs, especialmente, nas regiões de altitude do Sul do país, principalmente na região Serrana de Santa Catarina (SC) e nos Campos de Cima da Serra do Rio Grande do Sul (RS), para fins de comparação, a presente revisão objetivou reportar as principais cultivares e porta-enxertos de macieira e o manejo da adubação e irrigação e suas atualizações em pomares da Itália.

TABELA 1: Área e produção anual de maçãs nas diferentes regiões italianas.

\begin{tabular}{|c|c|c|}
\hline Região & $\begin{array}{c}\text { Área } \\
--- \text { ha --- }\end{array}$ & $\begin{array}{c}\text { Produção } \\
-- \text { (mil.t) ---- }\end{array}$ \\
\hline Piemonte & 5.465 & 156,9 \\
\hline Valle d'Aosta & 420 & 3,5 \\
\hline Lombardia & 1.992 & 51,1 \\
\hline Trentino Alto Adige & 28.150 & $1.396,8$ \\
\hline Veneto & 7.407 & 236,1 \\
\hline Friuli Venezia Giulia & 1.343 & 46,5 \\
\hline Liguria & 88 & 0,7 \\
\hline Emilia-Romagna & 6.121 & 162,3 \\
\hline Toscana & 718 & 12,3 \\
\hline Umbria & 253 & 5,3 \\
\hline Marche & 548 & 0,7 \\
\hline Lazio & 633 & 11,3 \\
\hline Abruzzo & 595 & 14,2 \\
\hline Molise & 430 & 3,4 \\
\hline Campania & 3.654 & 66,2 \\
\hline Puglia & 271 & 4,8 \\
\hline Basilicata & 353 & 7,7 \\
\hline Calabria & 490 & 5,3 \\
\hline Sicilia & 840 & 14,6 \\
\hline Sardegna & 393 & 3,9 \\
\hline Total & 60.164 & $2.210,4$ \\
\hline
\end{tabular}

Fonte: ISTAT (2007).

\section{Desenvolvimento}

\section{Cultivares e porta-enxertos}

As variedades mais utilizadas nos pomares italianos são a Gala, a Golden Delicious e a Fuji, representando, aproximadamente, $80 \%$ da maçã nacional (Tabela 2). Outras cultivares utilizadas são Braeburn e a Pink Lady®. O grupo das Galas domina o segmento de maçãs de maturação estival, sendo o segundo grupo varietal mais importante no país (Tabela 3). Entretanto, nos últimos anos se tem verificado uma diminuição da área cultivada com essa variedade, por causa da dificuldade em produzir frutos de coloração vermelha adequada, especialmente em pomares localizados em planícies. Por outro lado, tem se verificado aumento da área cultivada com o clone Galaxy, seleção clonal Evolution e Selecta, pertencente à variedade Gala, porque produzem frutos de coloração uniforme. Dentre as novidades de cultivares de maturação outonal se destaca a Modì (CIV G198*, CIV Ferrara), cultivar resistente à sarna da macieira (Venturia inaequalis Cooke G. Wint), com alta produtividade e frutos de coloração vermelho intenso, diâmetro grande, uniformes e com capacidade de suportar o armazenamento em atmosfera modificada durante longo período. Convém destacar que a variedade Ambrosia, também de maturação outonal, nos últimos anos tem sido cultivada com maior intensidade porque produz frutos de boa coloração, com excelente aparência e com composição química desejada (BERGAMASCHI; FAEDI, 2007; MISSERE et al., 2008).

No grupo varietal Red Delicious, de maturação outonal, as áreas cultivadas com plantas do clone Superchief® estão sendo substituídas por plantas clonais da histórica Red Chief ${ }^{\circledR}$. Já, do grupo varietal Fuji, de maturação hibernal, certamente um dos mais prolíficos no número de clones selecionados e introduzidos nos últimos anos na Itália, existe a Naga Fu 12® e a mais recente Zhen Aztec, adaptadas às regiões de planícies. Já os clones Kiku 8® e Raku Raku, de frutos estriados, são indicados para as áreas de montanha. Convém destacar que atualmente nesse grupo varietal tem sido observado aumento da área cultivada com plantas clonais Fubrax, com frutos estriados (BERGAMASCHI; FAEDI, 2007; MISSERE et al., 2008). A variedade Pink Lady ${ }^{\circledR}$, também de maturação hibernal é uma cultivar que se adapta as condições de solo e clima de planície, porém os seus frutos apresentam curta longevidade em pós-colheita (MISSERE et al., 2008). 
TABELA 2: Produção de maçã na Itália entre 2007 e 2011 (Dados em toneladas).

\begin{tabular}{|c|c|c|c|c|c|}
\hline \multirow{2}{*}{ Cultivar } & \multicolumn{5}{|c|}{ Ano agrícola } \\
\hline & 2007 & 2008 & 2009 & 2010 & 2011 \\
\hline Golden Delicious & 998.466 & 980.865 & 978.984 & 974.324 & 992.611 \\
\hline Red Delicious & 246.826 & 243.202 & 269.142 & 244.500 & 242.171 \\
\hline Imperatore & 91.182 & 91.179 & 66.877 & 81.495 & 77.160 \\
\hline Stayman & 20.093 & 18.485 & 15.553 & 18.037 & 16.544 \\
\hline Gala & 282.140 & 298.540 & 324.331 & 301.798 & 288.431 \\
\hline Granny & 98.320 & 99.194 & 114.500 & 113.376 & 105.819 \\
\hline Gloster & 580 & 530 & 419 & 300 & 293 \\
\hline Elstar & 1.820 & 1.493 & 1.137 & 931 & 1.112 \\
\hline Annurca & 45.000 & 45.000 & 35.000 & 35.000 & 40.024 \\
\hline Renette & 27.220 & 22.550 & 25.145 & 27.218 & 23.775 \\
\hline Jonagold & 29.029 & 24.474 & 23.868 & 14.357 & 17.626 \\
\hline Jonathan & 532 & 264 & 300 & 222 & 210 \\
\hline Braeburn & 90.793 & 86.666 & 96.510 & 96.607 & 96.073 \\
\hline Idared & 5.124 & 4.586 & 4.311 & 3.632 & 3.640 \\
\hline Fuji & 145.553 & 142.051 & 154.112 & 162.744 & 164.394 \\
\hline Cripps Pink & 65.590 & 58.190 & 80.323 & 70.481 & 74.850 \\
\hline Outras cultivares & 48.374 & 47.412 & 46.680 & 61.594 & 67.587 \\
\hline Total & 2.196 .643 & 2.164 .681 & 2.237.279 & 2.179 .615 & 2.212.320 \\
\hline
\end{tabular}

Fonte: Istat, 2012

TABELA 3: Épocas de maturação dos frutos, variedades e clones de macieira usados na Itália.

\begin{tabular}{|c|c|c|}
\hline $\begin{array}{l}\text { Estação de } \\
\text { maturação } \\
\text { dos frutos }\end{array}$ & Variedade & Clones \\
\hline Verão & Mondial Gala & $\begin{array}{c}\text { Baigent* Brookfield Gala }{ }^{\circledR}, \text { Gala Schnitzer* Schniga }{ }^{\circledR} \text {, Galaxy }\left(\text { Evolution }{ }^{\circledR} \text {, }\right. \\
\left.\text { Selecta }{ }^{\circledR}\right) \text {, Annaglo*, Obrogala* Delbard Gala }{ }^{\circledR} \text {, Gala Simmons* Bucheye }{ }^{\circledR} \text {, Gala } \\
\text { Rossa* Ruby Gala }{ }^{\circledR} \text {, Dalitoga }{ }^{\circledR}\end{array}$ \\
\hline Verão & Civni* Rubens ${ }^{\circledR}$ & - \\
\hline Verão & Dalinbel* Antares ${ }^{\circledR}$ & - \\
\hline Outono & Red Delicious & $\begin{array}{c}\text { Sandidge* Superchief }{ }^{\circledR}, \text { Camspur* Red Chief }{ }^{\circledR}, \text { Red Delicious* IT®, Valtod* Red } \\
\text { Cap® }{ }^{\circledR} \text {, Redkan*, Jéromine* }\end{array}$ \\
\hline Outono & Golden Delicious & $\begin{array}{c}\text { Leratess* Pink Gold } \AA, 1400 \text { KE* Goldrosiò }^{\circledR} \text {, Quémoni* Rosagold } \AA \text { Golden Delicious } \\
\text { cl. B*, Smoothee }{ }^{\circledR} \text {, Gold Chief }{ }^{\circledR} \text {, Gold Pink }{ }^{\circledR}\end{array}$ \\
\hline Outono & Milwa* Diwa ${ }^{\circledR} /$ Junami ${ }^{\circledR}$ & - \\
\hline Outono & Roho 3615* Evelina ${ }^{\circledR}$ & - \\
\hline Outono & Dalirail* & - \\
\hline Outono & Dalinip* & - \\
\hline Outono & CIV G 198* Modi® & - \\
\hline Outono & Coop39* Crimson Crisp ${ }^{\circledR}$ & - \\
\hline Outono & Nicoter* Kanzi ${ }^{\circledR}$ & - \\
\hline Outono & La Flamboyante* Mairac ${ }^{\circledR}$ & - \\
\hline Outono & Ambrosia* & - \\
\hline Inverno & Fuji & $\begin{array}{l}\text { Brak* Fuji Kiku } 8 \circledR \text {, Fuji Raku-Raku, ROFM811* Rubinfuji }{ }^{\circledR} \text {, Fubrax* Fuji KIKU®, } \\
\text { Fuji Toshiro }{ }^{\circledR} \text {, Aztec* Fuji Zhen }{ }^{\circledR} \text {, Fiero* September Wonder }{ }^{\circledR} \text {, Naga Fu12 }{ }^{\circledR}\end{array}$ \\
\hline Inverno & Braeburn & 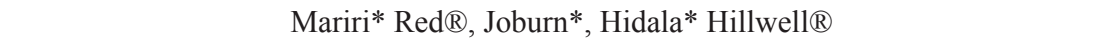 \\
\hline Inverno & Forlady* & - \\
\hline Inverno & Dalinette* Choupette® & - \\
\hline Inverno & Cripps Pink* Pink Lady ${ }^{\circledR}$ & Rosy Glow ${ }^{\circledR}$ \\
\hline
\end{tabular}

$*$ = nome da variedade patenteada e geneticamente protegida; $\mathbb{R}=$ nome comercial.

Fonte: Adaptado de Bergamaschi e Faedi (2007) e Missere et al. (2008). 
O porta-enxerto clonal mais usado nos pomares da Itália é o M9 (Malus pumila paradisiaca), clone T337. Isso porque, ele apresenta uma alta afinidade com a maior parte das cultivares, diminuindo o vigor da copa das plantas e facilitando as práticas culturais, como a poda, entre outras. Além disso, possui grande quantidade de raízes, estimulando a absorção de água e nutrientes e, por consequência, o crescimento vegetativo e o início de produção. Outros porta-enxertos mais vigorosos são normalmente utilizados em cultivares menos vigorosas ou quando as condições de clima e atributos de solo são desfavoráveis ao crescimento de plantas. Um porta-enxerto menos vigoroso que o M9 é o P16, que se adapta bem em condições de solos com alta fertilidade natural, porém é pouco difundido pela dificuldade de multiplicação nos viveiros (MISSERE et al., 2008).

\section{Manejo da adubação em pomares de macieira}

Os pomares de macieira são instalados em solos de textura média, com boa fertilidade natural, profundos e bem drenados. Na maioria das situações a calagem é dispensada, pois os solos são derivados de rochas calcárias e são ricos em Ca. Já a adubação pressupõe o fornecimento do nutriente quando o solo não o possui em quantidade suficiente para suprir a demanda da planta. Assim, a necessidade e a dose do nutriente a ser aplicado são definidas com base na análise de solo e análise foliar, sendo considerado o crescimento vegetativo. A dose do nutriente fornecida respeita a necessidade da planta, para a obtenção de produções satisfatórias, além de frutos de qualidade desejada, a fim de evitar perdas de nutrientes para espelhos de águas superficiais e lençóis freáticos, o que é constantemente acompanhado pelos órgãos ambientais (NEILSEN et al., 1995; TAGLIAVINI et al., 2007).

Os resultados de análise do solo, mas também de análise de tecido são interpretados com base em faixas de valores padrões. Exemplo disso podem ser os resultados de análise foliar encontrados na Tabela 4, onde a partir dos quais é possível estimar o estado nutricional das cultivares Golden Delicious, Braeburn, Fuji, Starking Delicious e Idared, na região do Trentino-Alto Adige (AICHNER; STIMPFL, 2002).
A adubação de pré-plantio é uma prática comum nos pomares. Os dejetos de animais são usados como fonte de nutrientes, sendo as quantidades definidas de acordo com a disponibilidade de nutrientes no solo, que é prescrita pela análise de solo. Os dejetos podem ser aplicados e incorporados na área total do pomar ou somente na linha de plantio. Alternativamente, podem ser usados compostos (ex. biossólidos) que, em geral, apresentam alta concentração de matéria seca e nitrogênio e menor relação $\mathrm{C} / \mathrm{N}$, mas às vezes podem conter altas concentrações de metais de transição, como o cobre, entre outros (TOSELLI et al., 2004).

TABELA 4: Faixas de concentração de nutrientes adequadas em folhas inteiras, coletadas no mês de julho, em cultivares Golden Delicious, Braeburn, Fuji, Starking Delicious e Idared, na região do Trentino-Alto Adige.

\begin{tabular}{|c|c|}
\hline \multirow{2}{*}{ Nutriente } & Teor do nutriente \\
\hline & $---\%$ \\
\hline Nitrogênio & $2,30-2,60$ \\
\hline Fósforo & $0,16-0,26$ \\
\hline Potássio & $1,20-1,70$ \\
\hline Cálcio & $1,20-2,00$ \\
\hline \multirow[t]{2}{*}{ Magnésio } & $0,20-0,36$ \\
\hline & --mg.kg-1-- \\
\hline Boro & $30-50$ \\
\hline Ferro & $75-115$ \\
\hline Manganês & $40-100$ \\
\hline Cobre & $5-12$ \\
\hline Zinco & $20-50$ \\
\hline
\end{tabular}

Fonte: Adaptado de Aichner e Stimpfl (2002).

As adubações de crescimento e de manutenção, na maioria das vezes são realizadas via fertirrigação, aproveitando a necessidade de suplementação de água, já que nas regiões produtoras de maça as chuvas são insuficientes para suprir a necessidade de água da planta em todo o ciclo. Os nutrientes são aplicados como sais solúveis, sendo a irrigação realizada em zona próxima ao caule e em doses fracionadas em intervalos regulares, o que permite maior recuperação dos nutrientes pelas plantas e menores transferências para os recursos naturais, como as águas (SORRENTI et al., 2005). A fertirrigação é iniciada na pré-floração, com pequenas doses, por exemplo, $3-5 \mathrm{~kg}$ de N.ha-1 $(=1-2 \mathrm{~g}$ de N.planta $\left.{ }^{-1}\right)$, e acontece, normalmente, em intervalos regulares de 7 a 10 dias até o final do verão (Tabela 5). 
Desde que as quantidades adicionadas sejam pequenas, o aporte de $\mathrm{N}$ via fertirrigação pode continuar em póscolheita, aumentando a disponibilidade do nutriente no solo e, hipoteticamente, a absorção, transporte e acumulação deles nos órgãos perenes.

TABELA 5: Plano de fertirrigação recomendado para um pomar de macieira na região da EmiliaRomagna, com densidade de 3000 plantas.ha ${ }^{1}$.

\begin{tabular}{ccccc}
\hline \begin{tabular}{c} 
Semana depois $\begin{array}{c}\text { Na plena } \\
\text { floração }\end{array}$ \\
\cline { 2 - 5 }
\end{tabular} & Nitrogênio & Fósforo & Potássio & Magnésio \\
\hline-1 & 0,5 & 0,8 & 0,0 & 0,0 \\
0 & 0,5 & 0,8 & 0,0 & 0,0 \\
1 & 0,5 & 0,4 & 0,0 & 0,0 \\
2 & 0,5 & 0,4 & 0,0 & 0,0 \\
3 & 1,0 & 0,4 & 0,0 & 0,0 \\
4 & 1,0 & 0,4 & 0,5 & 0,0 \\
5 & 1,5 & 0,2 & 1,0 & 0,5 \\
6 & 1,5 & 0,2 & 1,0 & 0,5 \\
7 & 1,9 & 0,2 & 1,0 & 0,5 \\
8 & 1,9 & 0,2 & 1,0 & 0,5 \\
9 & 1,9 & 0,2 & 1,0 & 0,5 \\
10 & 1,9 & 0,2 & 1,0 & 0,5 \\
11 & 1,0 & 0,2 & 2,0 & 0,0 \\
12 & 1,0 & 0,2 & 2,0 & 0,0 \\
13 & 1,0 & 0,2 & 2,0 & 0,0 \\
14 & 1,0 & 0,2 & 2,0 & 0,0 \\
15 & 0,5 & 0,1 & 2,5 & 0,0 \\
16 & 0,5 & 0,1 & 2,5 & 0,0 \\
17 & 0,5 & 0,1 & 2,5 & 0,0 \\
18 & 0,5 & 0,1 & 2,5 & 0,0 \\
\hline Total & $\mathbf{2 0 , 6}$ & $\mathbf{5 , 6}$ & $\mathbf{2 4 , 5}$ & $\mathbf{3 , 0}$ \\
\hline
\end{tabular}

Fonte: Scudellari e Tagliavini (1998).

O N é nutriente exportado em quantidades elevadas via fruto, comparativamente aos demais nutrientes requeridos pela planta (Tabela 6). Ele quando usado em solos com baixos teores de matéria orgânica pode aumentar a produção e alterar a qualidade de frutos, porém deve ser usado de acordo com os critérios de predição de adubação (ex.: análise de solo, análise de tecido, etc...), porque em doses elevadas e aplicadas sem critérios técnicos pode, por exemplo, retardar a maturação, devido à persistência da cor verde dos frutos, decorrência da clorofila e diluir a o cálcio no fruto, potencializando a ocorrência de distúrbios fisiológicos, como o bitter bit, entre outros (HIMELRICK; MCDUFFIE, 1983; CONWAY et al., 2002). Resultados de trabalhos mostram que o uso de sais de potássio via fertirrigação e ao longo do ciclo da macieira aumentam a disponibilidade do nutriente no solo, extraído pelo método de mineralização ácida, e os valores de acidez e a coloração vermelha de frutos, como os da cultivar Mondial Gala (MALAGUTI et al., 2006).

TABELA 6: Exportação de nutrientes em pomares de macieiras em produção.

\begin{tabular}{|c|c|c|}
\hline \multirow{2}{*}{ Nutriente } & Exportação $^{(1)}$ & Exportação via fruto $^{(2)}$ \\
\hline & ------ kg.ha-1 ------ & ---- kg.t ${ }^{-1}$ de frutos ---- \\
\hline Nitrogênio & $90-100$ & $0,50-0,60$ \\
\hline Fósforo & $10-20$ & $0,07-0,13$ \\
\hline Potássio & $115-150$ & 1,14 \\
\hline Cálcio & $130-140$ & 0,07 \\
\hline Magnésio & $15-25$ & $0,05-0,07$ \\
\hline
\end{tabular}

${ }^{(1)}$ Fonte: Marangoni et al. (1997); ${ }^{(2)}$ Fonte: Tagliavini et al. (2000).

$\mathrm{Na}$ Itália o Ca é usado em aplicações direcionadas ao fruto e realizadas frequentemente ao longo do ciclo da macieira, especialmente, naquelas cultivares, que são sensíveis a distúrbios fisiológicos, como a Jonagold, a Braeburn e a Fuji, (ex. "bitter bit" e "cook spot", entre outros). Nessas cultivares, trabalhos mostram que o $\mathrm{Ca}$ deixa de ser acumulado nos frutos algumas semanas antes da colheita. Porém, isso não é verificado em frutos de outras cultivares como na Golden Delicious, onde o acúmulo da $\mathrm{Ca}$ no fruto continua até a sua colheita (ZAVALLONI et al., 2001). Em cultivares pouco sensíveis aos distúrbios fisiológicos, como a Gala e Rome Beauty são recomendadas duas ou três aplicações de $\mathrm{Ca}$ direcionadas ao fruto, podendo ser na forma de $\mathrm{CaCl}_{2}$, em concentrações variando de 0,1 a $0,6 \mathrm{~g} \cdot \mathrm{L}^{-1}$. Entretanto, para cultivares sensíveis, se recomenda seis a oito aplicações ao longo do ciclo, com carência de dez dias entre cada uma delas. Resultados de trabalhos mostram que além do $\mathrm{CaCl}_{2}$ outras fontes de Ca como Ca complexado com peptídeos derivados de subprodutos da lã têm aumentado o teor do nutriente em frutos de Golden Delicious (MARCOLINI et al., 2004). Além dessa fonte, o gluconato de Ca, sal de Ca derivado do ácido glucônico, de recente introdução na agricultura, também tem causado aumento do nutriente nos frutos e esses, quando armazenados em atmosfera modificada, apresentam baixa incidência de Monilia, Gleosporium e Penicillium, comparativamente a outras fontes do nutriente (PORRO et al., 2007). 
Em pomares de Golden Delicious, Gala e Fuji exertadas sobre porta-enxertos vigorosos, entre os quais M11 e MM106, quando em solos calcários, com presença de elevadas concentrações de $\mathrm{Ca}$ e $\mathrm{Mg}$, tem sido observados sintomas visuais de deficiência de Mn nas folhas (PORRO et al., 2002). Nesses casos, aplicações foliares de Mn durante a primavera, usando como fonte o sulfato ou complexado de $\mathrm{Mn}$ e em doses variando de 0,2 a $0,5 \mathrm{~g} . \mathrm{L}^{-1}$ de $\mathrm{Mn}$ foram eficientes em aumentar o teor do nutriente na folha, sendo verificado efeito positivo na cor de fundo dos frutos.

\section{Manejo da irrigação e gestão da água em pomares de macieira}

A maior parte dos pomares de macieira instalados na Itália são irrigados, especialmente aqueles formados com porta-enxertos de fraco a médio vigor. A progressiva diminuição dos volumes de precipitação nas tradicionais regiões produtoras de maçã do país, associado ao aumento da temperatura média anual e somado a outros fatores, tem tornado a irrigação uma prática imprescindível para a obtenção de produtividades satisfatórias e frutos de qualidade (MANNINI, 2008).

O sistema de irrigação mais usado nos pomares é a microirrigação, gotejamento ou microaspersão, associada ou não a fertirrigação. Porém, em determinados pomares, como os localizados na região do Trentino-Alto Adige, norte do País, a irrigação por aspersão sobre a copa das plantas é usada como estratégia para defesa anti-gelo em geadas tardias (Figura 2a b). Em geral, essa irrigação é realizada quando a temperatura do ar, medida a 30 a $40 \mathrm{~cm}$ do solo é de, aproximadamente, $1^{\circ} \mathrm{C}$. Com a irrigação é preservada a integridade dos tecidos das plantas, mesmo quando a temperatura do ar for menor que $0^{\circ} \mathrm{C}$ (THALHEIMER; PAOLI, 2008). Em alguns pomares tem sido usado um sistema de irrigação duplo: o gotejamento para irrigação na linha e a aspersão para defesa anti-gelo.

A crescente necessidade de economia de água tem motivado a prática de irrigação mais racional, na tentativa de maximizar a eficiência da água distribuída. Para tal propósito, uma série de trabalhos, conduzidos em diversos pomares, com diferentes porta-enxertos tem sido realizados para estudar as técnicas de irrigação mais racionais e seu emprego otimizado (MANNINI, 2008). As informações obtidas nesses estudos permitiram a criação de sistemas de alerta para os fruticultores, como o obtido em $<$ http://irrigation.altavia.eu/>, onde é transmitido, via online ou telefone, sugestões de turnos e volumes de irrigação a serem realizados no pomar. Isso tem diminuído os volumes de água de irrigação usados e os custos de produção, sem prejudicar a produção e a qualidade dos frutos (GHINASSI et al., 2007; MANNINI, 2008).

A macieira, como em outras frutíferas, se adapta à irrigação com estresse hídrico controlado. Testes

FIGURA 2: Irrigação sobre a copa das plantas utilizada para a defesa das geadas na primavera em pomares de macieira (a) e na planta (b), na região Trentino-Alto Adige.
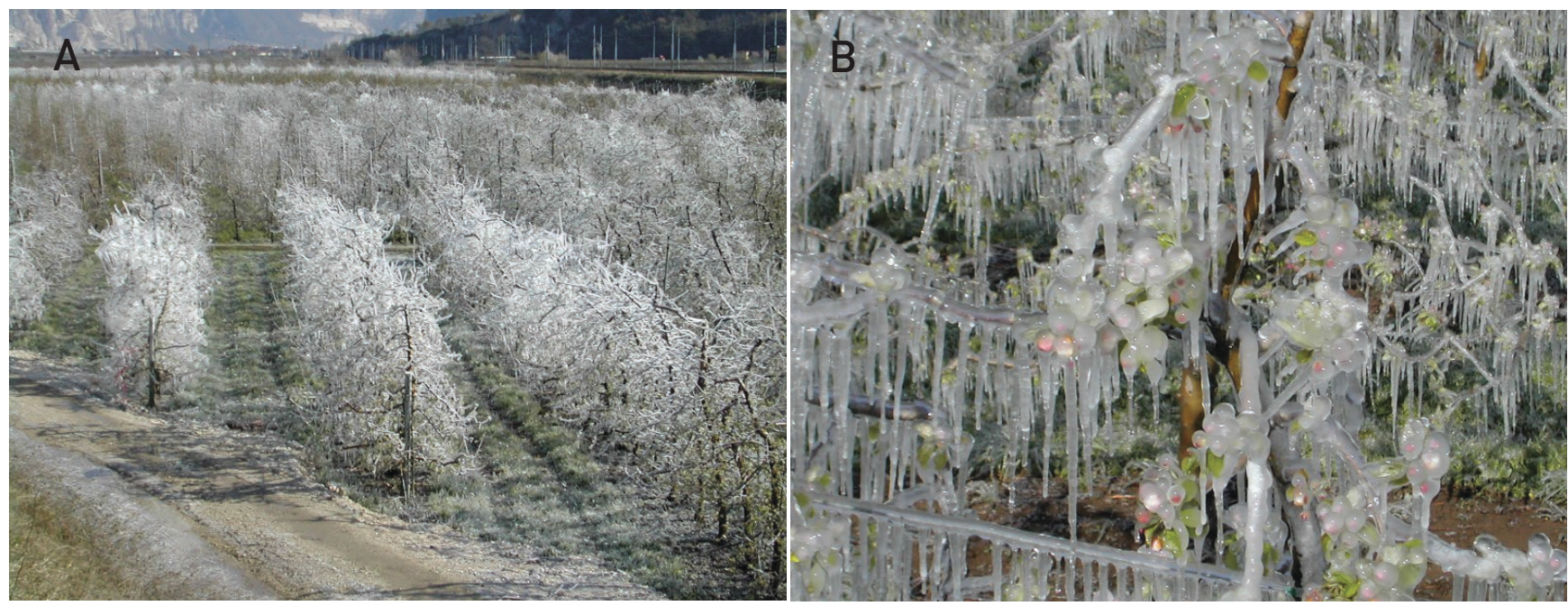
trienais realizados em pomares adultos das cultivares Gala, Fuji e Pink Lady ${ }^{\circledR}$ mostraram que a técnica do estresse hídrico controlado pode economizar de 15 a $60 \%$ da evapotranspiração máxima (Etm), sem afetar a produção e limitando o vigor das plantas (ANCONELLI et al., 2008).

Em ambientes de baixa disponibilidade hídrica para a irrigação de pomares, como por exemplo, a parte meridional da Itália, visando aumentar a eficiência do uso da água, tem sido usado a técnica de irrigação parcial na zona radicular das plantas. Nessa, metade das raízes das plantas recebem o volume de irrigação reduzido a 50\% e o restante das raízes não é irrigada. Alguns trabalhos realizados na Sicília em pomares com diversas cultivares como a Gala, a Golden Delicious, a Fuji e a Pink Lady ${ }^{\circledR}$, enxertadas sobre porta-enxertos de diferentes vigores, têm mostrado que a irrigação parcial na zona radicular das plantas diminui a condutância estomática, número de frutos, carga produtiva e a relação fruto/folha, comparativamente, às plantas irrigadas em sistemas convencionais (TALLUTO; LO BIANCO, 2007).

Os programas de melhoramento genético na Itália selecionaram cultivares e porta-enxertos de macieira que permitem a obtenção de altas produtividades e frutos de qualidade exigida pelo mercado consumidor. No manejo da adubação e irrigação, os nutrientes e a água tem sido fornecidos em quantidades próximas a real necessidade das plantas, permitindo adequada nutrição, produção satisfatória, frutos de qualidade e, evitando, sempre que possível, as perdas de nutrientes e água no ambiente.

\section{Referências}

AICHNER, M.; STIMPFL, E. Seasonal pattern and interpretation of mineral nutrient concentrations in apple leaves. Acta Horticulturae, Amsterdam, v. 594, p. 377-382, 2002.

ANCONELLI, S.; MANNINI, P.; SOLIMANDO, D.; GUIDOBONI, G. Effetti della gestione irrigua sul melo tramite stress idrico controllato. Rivista di Frutticoltura, Bologna, v. 70, n. 5, p. 52-59, 2008.

BERGAMASCHI, M.; FAEDI, W. Moderni orientamenti per la scelta varietale del melo. Rivista di Frutticoltura, Bologna, v. 9, p. 30-34, 2007.

CONWAY, W. S.; SAMS, C. E.; HICKEY, K. D. Pre-and postharvest calcium treatment of apple fruit and its effect on quality. Acta Horticulturae, Amsterdam, v. 594, p. 413-419, 2002.
DALPIAZ, A. Calo della produzione europea aiuterà il mercato del prodotto italiano. Rivista di Frutticoltura, Bologna, v. 10, p. 6-8, 2007.

FERRANDINO, A.; THEDY, L.; DUVERNEY, C.; STEFANO, R. Valutazione del quadro aromatico di mele Golden Delicious provenienti da diversi areali di coltivazione. Rivista di Frutticoltura, Bologna, v. 10, p. 39-44, 1999.

GHINASSI, G.; GIACOMIN, A.; IZZI, G. Irrigazione su base tensiometrica per un più razionale impiego dell'acqua. Rivista di Frutticoltura, Bologna, v. 10, p. 60-64, 2007.

HIMELRICK, D. G.; MCDUFFIE, R. F. The calcium cycle: uptake and distribution in apple trees. HortScience, Alexandria, v. 181, p. 147-151, 1983.

ISTAT - ISTITUTO DI STATISTICA ITALIANA. Superficie e produzione annua di mele nelle differenti Regioni Italiane. 2007. Disponível em: <http://www.istat.it/>. Acesso em: 20 out. 2009.

ISTAT - ISTITUTO DI STATISTICA ITALIANA. Superficie e produzione annua di mele nelle differenti Regioni Italiane. 2012. Disponível em: <http://www.istat.it/>. Acesso em: 10 jul. 2012.

LA IACONA, T.; GIACALONE, G. Caratterizzazione sensoriale delle mele Golden Delicious provenienti da diversi areali di coltura piemontesi. Italus Hortus, Firenze, v. 5, n. 2, p. 118-122, 2006.

MALAGUTI, D.; ROMBOLÀ, A. D.; QUARTIERI, M.; MARANGONI, B.; TAGLIAVINI, M. Effects of the rate of nutrients by fertigation and broadcast application in "Gala" and "Fuji" apple. Acta Horticulturae, Amsterdam, v. 721, n. 1, p. 165$172,2006$.

MANNINI, P. I fabbisogni idrici della frutticoltura emilianoromagnola nel Piano regionale di tutela delle acque. Rivista di Frutticoltura, Bologna, v. 5, p. 24-31, 2008.

MARANGONI, B.; TAGLIAVINI, M.; SCUDELLARI, D.; FERRARO, R. Aspetti tecnici della nutrizione minerale e dell'irrigazione nella moderna melicoltura. In: CONVEGNO DI VERONA "Melicoltura di pianura: quale futuro", 1, 1997, Verona, Atti... Verona: Camera di Commercio Industria Artigianato e Agricoltura di Verona, 1997. p. 173-193.

MARCOLINI, G.; TOSELLI, M.; BORGHINI, M.; LUCCHI, A.; QUARTIERI, M.; BALDI, E.; MALAGUTI, D.; GESSA, C.; MARANGONI, B. Effetto del formulato e dell'epoca di somministrazione sull'assorbimento del calcio nei frutti di Golden Delicious. In: GIORNATE SCIENTIFICHE SOI, VII, 2004, Napoli. Atti... Napoli: Società Orticola Italiana, 2004. p. 393

MISSERE, D.; COLOMBO, R.; PIRAZZINI, P. Novità varietali in mostra. Rivista di Frutticoltura, Bologna, v. 4, p. 88-91, 2008.

NEILSEN, D.; PARCHOMCHUCK, P.; NEILSEN, G. H.; BERARD, R.; HAGUE, E. J. Leaf nutrition and soil nutrients are affected by irrigation frequency and method for NP-fertigated Gala apple. HortScience, Alexandria, v. 120, p. 971-976, 1995.

PORRO, D.; COMAI, M.; DORIGONI, A.; STEFANINI, M.; CESCHINI, A. Manganese foliar application to prevent leaf drop. Acta Horticulturae, Amsterdam, v. 594, p. 229-336, 2002.

PORRO, D.; DORIGATTI, C.; RAMPONI, M.; ZATELLI, A. Incidenza di diversi apporti di calcio sulla qualità dei frutti di melo. In: GIORNATE SCIENTIFICHE SOI, 8, 2007, Sassari. Riassunti... Firenze: Italus Hortus, v. 14 (2oㅡ supplemento), 2007. p. 368. 
SCUDELLARI, D.; TAGLIAVINI, M. Principi teorici ed applicativi per l'impiego della fertirrigazione nel frutteto. Supplemento Terra e Vita, Bologna, v. 13, p. 4-7, 1998.

SORRENTI, G.; QUARTIERI, M.; TAGLIAVINI, M. La fertirrigazione delle specie arboree da frutto. Phytomagazine, Speciale Fertirrigazione, Verona, v. 1, p. 51-62, 2005.

TALLUTO, G.; LO BIANCO, R. Tecniche di irrigazione controllata (Prd) per la coltivazione in aree a bassa disponibilità idrica. Rivista di Frutticoltura, Bologna, v. 69, n. 10, p. 18-21, 2007.

TAGLIAVINI, M.; QUARTIERI, M.; ROMBOLÀ, A. D.; ZAVALLONI, C.; MALAGUTI, D.; MARANGONI, B. Ripartizione degli elementi minerali nei frutti degli alberi decidui. Rivista di Frutticoltura, Bologna, v. 62, n. 1, p. 83-87, 2000.

TAGLIAVINI, M.; TONON, G.; SCANDELLARI, F.; QUIÑONES, A.; PALMIERI, S.; MENARBIN, G.; GIOACCHINI, P.; MASIA,
A. Nutrient recycling during the decomposition of apple leaves (Malus domestica) and mowed grasses in an orchard. Agriculture, Ecosystems and Environment, Amsterdam, v. 118, p. 191-200, 2007.

THALHEIMER, M.; PAOLI, N. La difesa dalle gelate tardive nel melo: irrigazione si ma con meno acqua. Rivista di Frutticoltura, Bologna, v. 5, p. 48-51, 2008.

TOSELLI, M.; BALDI, E.; MARCOLINI, G.; MARANGONI, B. La concimazione pre-impianto delle colture arboree da frutto. L'Informatore Agrario, Verona, v. 47, p. 51-54, 2004.

ZAVALLONI, C.; MARANGONI, B.; TAGLIAVINI, M.; SCUDELLARI, D. Dynamics of uptake of calcium, potassium and magnesium into apple fruit in a high density planting. Acta Horticulturae, Amsterdam, v. 564, p. 113-119, 2001. 